\title{
Flexible Needle Steering System for Percutaneous Access to Deep Zones of the Brain
}

\author{
J.A. Engh ${ }^{1,2}$, G. Podnar ${ }^{1}$, S.Y. Khoo ${ }^{1}$, and C.N. Riviere ${ }^{1}$ \\ ${ }^{1}$ The Robotics Institute, Carnegie Mellon University \\ ${ }^{2}$ Department of Neurological Surgery, University of Pittsburgh \\ Pittsburgh, PA 15213
}

\begin{abstract}
The authors describe a minimally invasive flexible needle-steering system to access deep areas of the brain. The design exploits the inherent bending of a beveled-tip needle when passing through tissue. Precise control of the rotation of the needle allows for an unlimited variety of trajectories. Potential advantages to this system include the possibility of catheter-based therapy delivery within the brain with minimal trauma to surrounding structures, and the ability to navigate around critical cerebral structures to reach deep zones within the brainstem, thalamus or other subcortical regions.
\end{abstract}

\section{INTRODUCTION}

Brain cancer is a devastating ailment with a scant proportion of long-term survivors. Over 13,000 Americans die from brain tumors each year, not including the many thousands who suffer intracranial complications of intracranial metastases [1]. These tumors tend to be poorly encapsulated, with blurred margins and microinvasion of the surrounding brain parenchyma [2]. As a result, surgical resection is generally not curative. The vast majority of these tumors recur locally, i.e. within $2 \mathrm{~cm}$ of the original resection cavity [3]. Despite the application of adjuvant treatments such as fractionated radiation and a multitude of chemotherapeutic agents, the disease remains generally incurable.

The blood-brain barrier is a critical obstacle to the delivery of systemic agents into the brain, and may be the major reason for the failure of systemic chemotherapy in the brain [4]. Since it restricts the access of larger molecules in particular, this barrier prevents the entry of targeted therapeutic molecules into the central nervous system (CNS) without significant systemic toxicity. As a result, great interest has developed in compartmental therapy for brain tumors [5]. This approach bypasses the blood-brain barrier via direct application of therapeutic agents into the CNS, either at the time of tumor removal or via percutaneous stereotactic delivery. However, at present, a straight path must be taken from the cortex to the desired target, and there is no ability to curve around critical structures or vessels. If the needle is offtarget or if multiple zones must be reached, an entirely new channel must be created. As a result, the risk of hemorrhage or neurologic injury is increased. In addition, there is no capability to safely manipulate an intracerebral catheter deep within a three-dimensional "treatment zone" itself, which might improve the precision, versatility and safety of local therapy for these lesions.

Funding provided by the Simeon M. Jones, Jr. and Katharine Reed Jones Fund and the Walter L. Copeland Fund of The Pittsburgh Foundation.

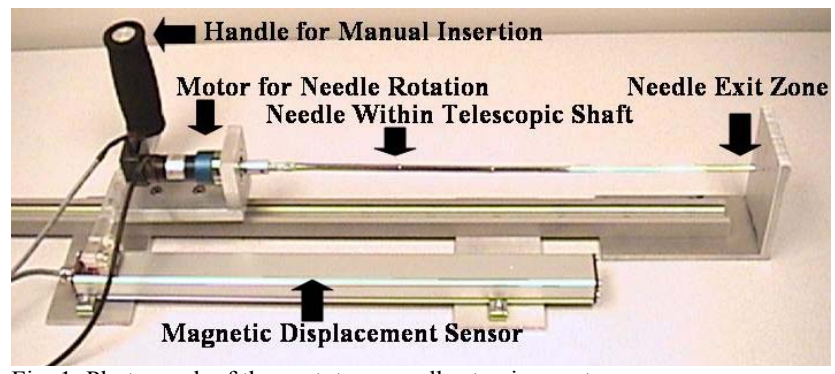

Fig. 1: Photograph of the prototype needle steering system.

Recent research has demonstrated that the tendency of a standard beveled-tip (Quincke) needle to bend during insertion can be exploited in order to steer the needle by rotation of its shaft $[6,7]$. The needle deflects in the direction of its sharpened tip, the curvature being a function of the stiffness of the needle relative to the surrounding tissue [8]. In this model, precise rotation of the needle provides directional control of the needle trajectory. The authors propose an adaptation to this system which augments the shaft-rotation approach by adding proportional control of needle deflection. The resultant precision and versatility is ideal for stereotactic navigation within the brain.

\section{DESIGN}

The first prototype of the needle-steering system is depicted in Figure 1. The handle allows the operator to advance the device into the tissue substrate. The electric motor beneath the handle rotates the needle shaft in the desired direction during insertion. The extended needle is kept within a telescoping conduit prior to insertion to prevent unwanted bending of the shaft. The magnetic linear displacement sensor at the bottom of the figure monitors the depth of insertion. A feedback loop from this sensor provides exact distance measurements to a computer. The computer then calculates the direction and degree of needle spinning, which it regulates via a connection to the motor.

Rather than control only the direction of needle bending, this device controls the degree of bending as well. The greatest degree of bending is achieved by no spin at all, allowing the inherent tendency of the needle to curve away from the bevel to be maximized. A straight trajectory is created by spinning the needle at a constant rate. Any trajectory in between these two can be achieved by "duty-cycling" the rotation of the needle, i.e. rotating the needle in a rapid "spin-stop-spin-stop" fashion such that the bevel stops in the same orientation each 


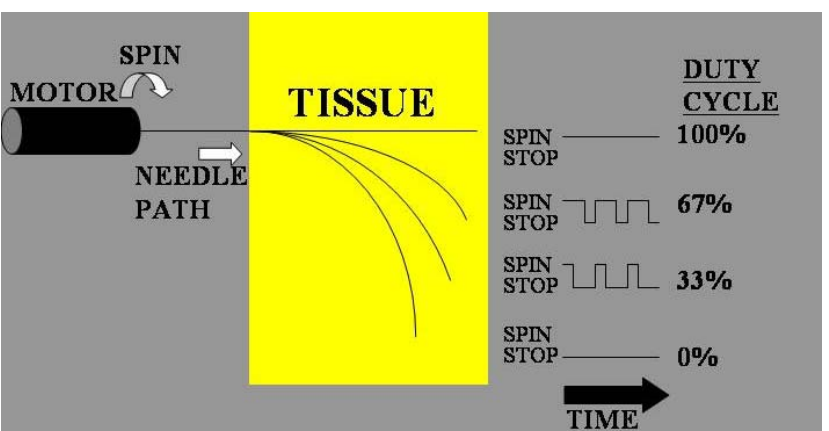

Figure 2: Conceptual diagram of trajectories of a flexible bevel-tipped needle created by "duty-cycling" the rotation of the needle. The uppermost trajectory is the straight trajectory created by a constant rate of spin. The second trajectory is a $67 \%$ duty cycle, i.e. spinning $2 / 3$ of the time. The third trajectory is a $33 \%$ duty cycle. The last trajectory results from no spinning at all, which creates the maximum possible bending of the needle.

time. Longer "stop" intervals create steeper curvature of the needle, and longer "spin" intervals create a straighter trajectory. The effect of these differing spin ratios is demonstrated in Figure 2.

\section{TESTING}

The prototype was tested in a phantom of Knox Gelatine (Kraft Foods Global, Inc., Tarrytown, NY) mixed at a ratio of $1.3 \mathrm{cc}$ of gelatin to $20 \mathrm{cc}$ of boiling water. This particular substrate has been shown to demonstrate a frictional force on a straight catheter which is within an order of magnitude of the frictional force provided by brain tissue in vitro [9]. The phantom was allowed to harden within a square plastic container over a 1 X $1 \mathrm{~cm}$ grid. A series of small holes were drilled into the side of the container, and an aluminum tube was inserted into each hole as an introducer device. A nitinol wire with a custom-beveled tip was inserted through the introducer device until the bevel could be visualized immediately above the grid matrix, pointing laterally. No introducer site was used twice.

The variable for this experiment was the duty cycle of needle spinning. For the first test, no spinning was performed $(0 \%$ duty cycle). For the second test, a $50 \%$ duty cycle at $2 \mathrm{~Hz}$ with equivalent spin/stop periods was performed. The final test utilized a $100 \%$ duty cycle, constantly spinning at $2 \mathrm{~Hz}$. Photographs were taken of all trajectories to demonstrate the degree of needle bending.

Figure 3: Preliminary testing of the device in a gelatin phantom. In each photograph, the needle tip is oriented toward the top of the page. The needle enters from the left, and the white arrow depicts the needle location beneath a cellophane grid. The figure demonstrates trajectories generated by (a) no spinning $(0 \%$ duty cycle); (b) equal intervals on \& off $(50 \%$ duty cycle); and (c) continuous spinning $(100 \%$ duty cycle). The needle in (c) is partially obscured by the overlying gridlines.
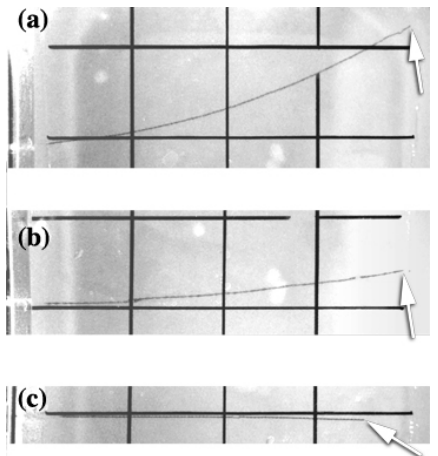

\section{DISCUSSION}

The ability to safely direct the path of a micro-needle into the brain has the potential to change the standard treatment paradigms for many human brain tumors. Using this device, the outer surface of a tumor might be accessed via a standard straight trajectory. Then, the micro-needle could be advanced to any desired spot within the lesion, a catheter would be passed over it, and any infusible catheter-based therapy could be delivered to the margin of the lesion, including toxins, chemotherapy, dendritic cells, stem cells, gene therapy, etc. The catheter would then be brought back to the margin of the lesion, and then advanced to another desired target, where more catheter-based therapy could be delivered. The process would be analogous to driving a car into a parking spot within a garage, then backing up outside the garage to the same starting point, then driving into a different spot within the garage, and so forth. Intraoperative computed tomography could feasibly be used for confirmation of accurate catheter placement prior to infusion.

Another possible application of such a system is deep brain stimulation, in which minute trajectory adjustments are often necessary to achieve appropriate electrode localization. Avoiding an entirely new path from the cortex to a deep nucleus could minimize risk in this situation. Other cerebral applications might include stereotactic lesioning for epilepsy and delivery of stem cells for neurodegenerative disorders.

The feasibility of proportional control of both the direction and the degree of bending of a beveled-tip needle within a tissue phantom has been demonstrated. Such a system would be ideal for versatile stereotactic intracerebral navigation. Future research will focus on maximizing the degree of needle bending through alterations in needle design, and making the device compatible with existing stereotactic frames.

\section{REFERENCES}

[1] S.H. Landis, T. Murray, S. Bolden and P.A. Wingo, "Cancer statistics, 1999," CA Cancer J. Clin. 49(1):8-31, 1999.

[2] C. Daumas-Duport and G. Szikla, "Delimitation et configuration spatiale des gliomes cerebraux [article in French]," Neurochirurgie 27(5):273284, 1981.

[3] I.F. Dunn and P.M. Black, "The neurosurgeon as local oncologist: cellular and molecular neurosurgery in malignant glioma therapy," Neurosurgery 52(6):1411-1422, 2003.

[4] E.M. Kemper, W. Boogerd, I. Thuis, J.H. Beijnen and O. Van Tellingen, "Modulation of the blood-brain barrier in oncology: therapeutic opportunities for the treatment of brain tumors," Cancer Treat. Rev. 30(5):415-423, 2004.

[5] M. Westphal and P.M. Black, "Perspectives of cellular and molecular neurosurgery," J. Neurooncol. 70(2):255-269, 2004.

[6] R.J. Webster III, N.J. Cowan, G. Chirikjian, and A.M. Okamura, "Nonholonomic modeling of needle steering," $9^{\text {th }}$ Int. Symp. Exp. Rob. June 2004.

[7] R.J. Webster III, J. Mernisevic, and A.M. Okamura, "Design considerations for robotic needle steering," IEEE Int. Conf. Rob. Auto. 3599-3605, 2005.

[8] H. Kataoka, T. Washio, M. Audette and K. Mizuhara, "A model for relations between needle deflection, force, and thickness on needle penetration," Lect. Notes Comput. Sci. 2208:966-974, 2001.

[9] R.C. Ritter, E.G. Quate, G.T. Gillies, M.S. Grady, M.A. Howard III, and W.M. Broaddus, "Measurement of friction on straight catheters in in vitro brain and phantom material," IEEE Trans. Biomed. Eng. 45(4):476-485, 1998 